\title{
THE ASYMPTOTIC EXPANSION FOR THE TRACE OF THE HEAT KERNEL ON A GENERALIZED SURFACE OF REVOLUTION
}

\author{
BY
}

PING-CHARNG LUE

\begin{abstract}
Let $M$ be a smooth compact Riemannian manifold without boundary. Let $I$ be an open interval. Let $h(r)$ be a smooth positive function. Let $g$ be the metric on $M$. Consider the fundamental solution $E\left(x, y, r_{1}, r_{2} ; t\right)$ of the heat equation on $M \times I$ with metric $h^{2}(r) g+d r \otimes d r$ (when $E$ exists globally we call it the heat kernel on $M \times I)$. The coefficients of the asymptotic expansion of the trace $E$ are studied and expressed in terms of corresponding coefficients on the basis $M$. It is fulfilled by means of constructing a parametrix for $E$ which is different from a parametrix in the standard form. One important result is that each of the former coefficients is a linear combination of the latter coefficients.
\end{abstract}

0. Introduction. We shall present here a relation between the coefficients of pointwise asymptotic expansion for the trace of the heat kernel on a generalized surface of revolution and the corresponding coefficients on its cross section. Let $(M, g)$ be a compact Riemannian manifold with metric $g$ and $I$ be an open interval, $h(r): I \rightarrow R^{+}$a smooth positive function. Then we call $M \times I$ with metric $h^{2}(r) g+$ $d r \otimes d r$ a generalized surface of revolution with section $M$. The asymptotic expansion for the heat kernel on $M$ had been introduced and studied by Minakshisundaram and Pleijel [7]. Then the first author in a short paper [8] gave another proof and wrote the expansion in the following form:

$$
E(x, x, t) \underset{t \rightarrow 0}{\sim} \frac{1}{(2 \sqrt{\pi})^{n}} t^{-n / 2}\left(1+a_{1}(x) t+a_{2}(x) t^{2}+\cdots\right) .
$$

Here $E(x, x, t)$ denotes the pointwise trace of the heat kernel on $M^{n}$. The heat kernel $E(x, y, t)$ can be written as $E(x, y, t)=\sum e^{-\lambda_{i} t} \phi_{i}(x) \phi_{i}(y)[1]$ where $0 \leqslant \lambda_{0} \leqslant$ $\lambda_{1} \cdots \uparrow \infty$ are the eigenvalues of the Laplacian on $M$ and $\left\{\phi_{i}\right\}$ are corresponding orthonormal eigenfunctions. If we integrate $(0,1)$ with respect to the volume form we obtain [1]

$$
\sum_{i=0}^{\infty} e^{-\lambda_{i} t} \underset{t \rightarrow 0}{\sim}(4 \pi t)^{-n / 2}\left(a_{0}+a_{1} t+a_{2} t^{2}+\cdots\right) .
$$

The coefficients $\left\{a_{i}\right\}$ have geometric meaning; for example $a_{0}$ is the volume of the manifold, and when $n=2, a_{1}=\pi \chi(M) / 3$ (where $\chi\left(M^{2}\right.$ ) is the Euler characteristic of $M^{2}$ ). While it is known that the $\left\{a_{i}\right\}$ depend on the curvature tensor $R$ and its

Received by the editors February 23, 1981 and, in revised form, June 8, 1981. The paper has been presented to the 772 special session of the AMS on Global Differential Geometry at Riverside, November 16-17, 1979.

1980 Mathematics Subject Classification. Primary 58G11; Secondary 34E05.

(c) 1982 American Mathematical Society 0002-9947/81/0000-0611/\$05.00 
successive covariant derivatives [1], it is very difficult to calculate them explicitly. In fact, only the first few of them have been calculated for general manifolds. For some special manifold, such as compact symmetric spaces of rank one, Cahn and Wolf [2] have found an explicit formula for all the coefficients.

The method used by Minakshisundaram-Pleijel [7] to study the asymptotic expansion was to construct a sufficiently good parametrix. The properties of the heat kernel at the diagonal are determined by the parametrix. Therefore it might appear that the most natural way of studying our problems would be to start with a parametrix of the following form:

$$
\frac{1}{(4 \pi t)^{(n+1) / 2}} e^{\rho^{2} / 4 t}\left(\bar{u}_{0}\left(r_{1}, r_{2}, x, y\right)+\bar{u}_{1}\left(r_{1}, r_{2}, x, y\right) t+\bar{u}_{2}\left(r_{1}, r_{2}, x, y\right) t^{2}+\cdots\right)
$$

Here $(x, y)$ denotes points on the cross section, $r_{1}, r_{2} \in R$ and $\rho$ denotes the distance between $\left(r_{1}, x\right)$ and $\left(r_{2}, y\right)$ on $M \times R$. But even though it is possible to express $\rho$ in terms of the distance function on $M$ and the warping function $h(r)$, this approach does not lead to a formula for the coefficients on $M \times I$ in terms of the corresponding coefficients on $M$ (see $\S I V)$. Instead we construct a parametrix of nonstandard form. The idea of constructing such a parametrix was initially suggested by the following considerations. By using separation of variables we can express the heat kernel on $M \times R$ in the form $\sum_{i=0}^{\infty} f_{i}\left(r_{1}, r_{2}, t\right) \phi_{i}(x) \phi_{i}(y)$. Then the Laplace transform of each $f_{i}$ will be a parametrix for the kernel of the resolvent of the associated 1-dimensional Sturm-Liouville problem. Gel'fand and Dikii have studied the asymptotic expansions for the trace of these kernels [4]. This leads to an asymptotic expansion for the $f_{i}$. The construction of our parametrix was motivated in part by a certain formal procedure for "adding" these asymptotic expansions. Here the following difficulty arises: the asymptotic expansion of each $f_{i}$ starts with the term $t^{-1 / 2}$. But the asymptotic expansion we want must start with $t^{-(n+1) / 2}$. In fact we have infinitely many asymptotic expansions starting with $t^{-1 / 2}$ and not holding uniformly. We want to "add" these in such a way to obtain an expansion starting with $t^{-(n+1) / 2}$.

In concluding this introduction, the author would like to thank his advisor, Jeff Cheeger, who patiently guided the work.

\section{Preliminaries.}

I.1. The Laplacian on $M \times I$. Let $M \times I$ be the generalized surface of revolution as we defined it in the introduction. If we denote the Laplacians on $M \times I$ and $M$ by $\Delta$ and $\underline{\Delta}$ respectively then a standard computation shows

\section{I.1.1. PROPOSITION.}

$$
\Delta=-\left(\frac{\partial}{\partial r}\right)^{2}-\frac{n h^{\prime}(r)}{h(r)} \frac{\partial}{\partial r}+\frac{1}{h^{2}(r)} \Delta .
$$


I.2. Asymptotic expansions. We will use the following result.

I.2.1. THEOREM. If $f(z)$ is analytic in $-\alpha<\theta<\alpha, 0<r<b, z=r e^{i \theta}$ and if $f(z) \underset{z \rightarrow 0}{\sim} \sum_{k=0}^{\infty} a_{k} z^{k}$ uniformly in $\theta$ then $f^{\prime}(z) \underset{z \rightarrow 0}{\sim} \sum_{k=1}^{\infty} k a_{k-1} z^{k-1}$ uniformly in any small sector $-\alpha<\alpha_{1}<\theta<\alpha_{2}<\alpha$.

Proof. See [6].

I.2.2. CoRollary. If $\sum_{i=0}^{\infty} e^{-\lambda_{i} t} \underset{t \rightarrow 0}{\sim} t^{-n / 2}\left(a_{0}+a_{1} t+\cdots\right)$ then

$$
\sum_{i=0}^{\infty} \lambda_{i} e^{-\lambda_{i} t} \underset{t \rightarrow 0}{\sim} \frac{n}{2} t^{-n / 2-1} a_{0}+\left(\frac{n}{2}-1\right) t^{-n / 2} a_{1}+\cdots .
$$

Proof. $\sum_{i=0}^{\infty} e^{-\lambda_{i} t}$ is analytic in $-\alpha<\theta<\alpha . \alpha<\pi / 2$ [1] so this follows from Theorem I.2.1.

We also need

I.2.3. THEOREM. Suppose $F(s)$ is the Laplace transform of $f(t)$ and $F(s)$ has a half-plane of convergence. If $f(t) \underset{t \rightarrow 0}{\sim} \sum_{\nu=0}^{\infty} c_{\nu} t^{\lambda \nu}$ (where $\left.-1<R \lambda_{0}<R \lambda_{1} \cdots\right)$ then

$$
F(s) \underset{s \rightarrow \infty}{\sim} \sum_{\nu=0}^{\infty} c_{\nu} \frac{\Gamma\left(\lambda_{\nu}+1\right)}{s^{\lambda_{\nu+1}}} .
$$

$R \lambda_{i}$ denotes the real part of $\lambda_{i}$.

Proof. See [9].

1.3. Heat kernels and their parametrices.

I.3.1. Definition. A fundamental solution of the heat equation on a smooth Riemannian manifold $N$ is defined as a function $F$ on $N \times N \times R_{+}^{*}$ which satisfies the following:

(1) $F$ is $C^{0}$ in the three variables, $C^{2}$ in the second variable, $C^{1}$ in the third variable.

(2) $\left(\Delta_{2}+\partial / \partial t\right) F=0$. Here $\Delta_{2}$ is the Laplacian applied to the second variable.

(3) $\lim _{t \rightarrow 0} F(x, \cdot, t)=\delta_{x}$ for any $x \in N$. $^{1}$

I.3.2. Definition. When $N$ is compact the fundamental solution is unique. We call it the heat kernel of $N$.

REMARK. Since we are interested in the case of pointwise asymptotic expansion, we can regard $M \times I$ as a portion of a larger compact smooth manifold.

I.3.3. Definition. We call $H$ a parametrix of $\square=\Delta+\partial / \partial t$ if it satisfies:

(i) $H \in C^{\infty}\left(N \times N \times R_{+}^{*}\right)$.

(ii) $\square H$ can be extended to become a function in $C^{0}\left(N \times N \times R_{+}\right)$.

(iii) $\lim _{t \rightarrow 0} H(x, \cdot, t)=\delta_{x}$ for all $x \in N$.

In the definition, $R_{+}$denotes the nonnegative real numbers, $R_{+}^{*}$ denotes the positive reals and $\delta_{x}$ denotes the Dirac function at the point $x$.

${ }^{1}$ For $R_{+}^{*}, \delta_{x}$ see Definition I.3.3. 
II. Construction of a parametrix. We will construct a parametrix of the following nonstandard form:

$$
\begin{array}{r}
\frac{1}{2 \sqrt{\pi}} \sum_{i=0}^{\infty} h^{-n / 2}\left(r_{1}\right) h^{-n / 2}\left(r_{2}\right) \exp \left(-\frac{\left(r_{1}-r_{2}\right)^{2}}{4 t}\right) \exp \left(-\frac{\lambda_{i} t}{h\left(r_{1}\right) h\left(r_{2}\right)}\right) \\
\cdot \sum_{j=0}^{\infty} a_{j}\left(r_{1}, r_{2}, \lambda_{i}\right) \phi_{i}(x) \phi_{i}(y) t^{j-1 / 2}
\end{array}
$$

Apply the heat operator $\square_{2}=\Delta_{2}+\partial / \partial t$ to the above expansion with respect to the second variable $\left(r_{2}, y\right)$. Collect those terms with the same power of $t$ and set the resulting expression equal to 0 . In particular by setting the coefficient of $t^{j-1 / 2}$ equal to zero we get

$$
\begin{gathered}
\left(u_{i}\left(r_{2}\right)-\frac{\lambda_{i}}{h\left(r_{1}\right) h\left(r_{2}\right)}\right) a_{j-1}-\left(\frac{\partial}{\partial r_{2}}\right)^{2} a_{j-1}-\left(r_{1}-r_{2}\right) \frac{\lambda_{i} h^{\prime}\left(r_{2}\right)}{h\left(r_{1}\right) h^{2}\left(r_{2}\right)} a_{j-1} \\
+j a_{j}-\left(r_{1}-r_{2}\right) \frac{\partial}{\partial r_{2}} a_{j}-\frac{\lambda_{i}\left(h\left(r_{2}\right) h^{\prime \prime}\left(r_{2}\right)-2\left(h^{\prime}\left(r_{2}\right)\right)^{2}\right)}{h\left(r_{1}\right) h^{3}\left(r_{2}\right)} a_{j-2} \\
-2 \frac{\lambda_{i} h^{\prime}\left(r_{2}\right)}{h\left(r_{1}\right) h^{2}\left(r_{2}\right)} \frac{\partial}{\partial r_{2}} a_{j-2}-\left(\frac{\lambda_{i} h^{\prime}(r)}{h\left(r_{1}\right) h^{2}(r)}\right)^{2} a_{j-3}=0
\end{gathered}
$$

where $j \geqslant 0, a_{-3}=a_{-2}=a_{-1}=0$.

$$
u_{i}(r)=\frac{n}{2}\left(\frac{n}{2}-1\right) \frac{h^{\prime}(r)^{2}}{h^{2}(r)}+\frac{n}{2} \frac{h^{\prime \prime}(r)}{h(r)}+\frac{\lambda_{i}}{h^{2}(r)}
$$

We can then solve $a_{j}$ successively: $a_{0}=$ const. Here we use the following normalizing conditions; let $a_{0}=1$.

$$
\begin{aligned}
a_{2}= & \frac{1}{2\left(r_{1}-r_{2}\right)^{2}}\left(\int_{r_{1}}^{r_{2}} u_{i}(r) d r\right)^{2}+\frac{1}{r_{1}-r_{2}}\left(\int_{r_{1}}^{r_{2}} u_{i}(r) d r\right) \frac{\lambda_{i}}{h\left(r_{1}\right) h\left(r_{2}\right)} \\
& -\frac{2}{\left(r_{1}-r_{2}\right)^{3}} \int_{r_{1}}^{r_{2}} u_{i}(r) d r-\frac{1}{\left(r_{1}-r_{2}\right)^{2}}\left(u_{i}\left(r_{1}\right)+u_{i}\left(r_{2}\right)\right)+\frac{1}{2} \frac{\lambda_{i}}{h\left(r_{1}\right) h\left(r_{2}\right)} .
\end{aligned}
$$


The lower limit is so chosen in order to avoid the singularities which would arise when $r_{1}=r_{2}$ if we did otherwise. In general,

$$
\begin{array}{r}
a_{j+1}=\frac{1}{\left(r_{1}-r_{2}\right)^{j+1}} \int_{r_{1}}^{r_{2}\{}-\left(r_{1}-r\right)^{j}\left(\frac{\lambda_{i} h^{\prime}(r)}{h\left(r_{1}\right) h(r)}\right)^{2}-2\left(r_{1}-r\right)^{j} \frac{\lambda_{i} h^{\prime}(r)}{h\left(r_{1}\right) h^{2}(r)} \\
\cdot \frac{\partial}{\partial r_{2}} a_{j-1}-\left(r_{1}-r\right)^{j} \frac{\lambda_{i}\left(h(r) h^{\prime \prime}(r)-2 h^{\prime}(r)\right)^{2}}{h\left(r_{1}\right) h^{3}(r)} a_{j-1} \\
-\left(r_{1}-r\right)^{j} \frac{\partial^{2}}{\partial r_{2}^{2}} a_{j}+\left(r_{1}-r\right)^{j}\left(u_{i}(r)-\frac{\lambda_{i}}{h\left(r_{1}\right) h(r)}\right) a_{j} \\
\left.-\left(r_{1}-r\right)^{j+1} \frac{\lambda_{i} h^{\prime}\left(r_{2}\right)}{h\left(r_{1}\right) h^{2}(r)} a_{j}\right\} d r .
\end{array}
$$

Let $H_{k}$ denote the expression (2.1) with the second summation replaced by the finite $\operatorname{sum} \Sigma_{j=0}^{k}$.

II.1. MAIN THEOREM. $H_{k}$ is a parametrix for $\square$ if: $\left[\frac{k}{3}\right]>\frac{n}{2}+\frac{3}{2}$. More precisely we multiply $H_{k}$ by a $C^{\infty}$ cut off function

$$
\eta_{R}\left(\rho\left(\left(r_{1}, x\right),\left(r_{2}, y_{2}\right)\right)\right)= \begin{cases}1, & \rho \leqslant R / 2, \\ 0, & \rho>R,\end{cases}
$$

in order to get a parametrix in the sense we have defined.

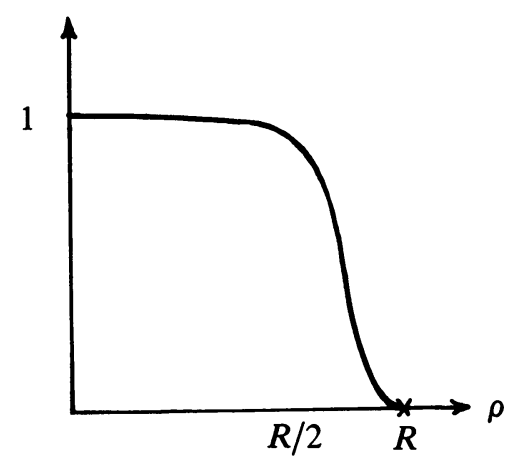

In order to prove the theorem we need to check the conditions for a parametrix (I.3.3). For the first condition we need the following lemma.

II.2. LEMMA. $a_{j+1}\left(r_{1}, r_{2}, \lambda_{i}\right)$ is $C^{\infty}$ in the variables $r_{1}, r_{2}$.

Proof. We prove it by induction. Write

$$
a_{j+1}=\frac{1}{\left(r_{1}-r_{2}\right)^{j+1}} \int_{r_{1}}^{r_{2}}\left(r_{1}-r\right)^{j} \mathcal{Q}_{j+1}\left(r_{1}, r, \lambda_{i}\right) d r .
$$


Here $\mathfrak{Q}_{j+1}$ is $C^{\infty}$ in $r_{1}, r_{2}$ from the induction hypothesis. In order to show that $a_{j+1}$ is $C^{\infty}$ it is then sufficient to check that $\left(\partial / \partial r_{1}\right)^{k}\left(\partial / \partial r_{2}\right)^{\prime} a_{j+1}$ exists for $r_{1}=r_{2}$. By Taylor's theorem

$$
\begin{aligned}
\mathbb{Q}_{j+1}\left(r_{1}, r, \lambda_{i}\right)= & \mathbb{Q}_{j+1}\left(r_{1}, r_{1}, \lambda_{i}\right)-\left(\partial / \partial r_{2}\right) \mathbb{Q}_{j+1}\left(r_{1}, r_{1}, \lambda_{i}\right)\left(r_{1}-r\right) \\
& +\cdots+\left((-1)^{k+l} /(k+l) !\right)\left(\partial / \partial r_{2}\right)^{k+l} \mathbb{Q}_{j+1}\left(r_{1}, \xi, \lambda_{i}\right)\left(r_{1}-r\right)^{k+l},
\end{aligned}
$$

$\xi$ is a number between $r_{1}$ and $r$. Therefore,

$$
\begin{aligned}
a_{j+1}= & \frac{\mathcal{Q}_{j+1}\left(r_{1}, r_{1}, \lambda_{i}\right)}{j+1}-\frac{\left(\partial / \partial r_{2}\right) \mathbb{Q}_{j+1}\left(r_{1}, r_{1}, \lambda_{i}\right)\left(r_{1}-r_{2}\right)}{j+2} \\
& +\cdots+\frac{(-1)^{k+l}}{\left(r_{1}-r_{2}\right)^{j+1}} \int_{r_{1}}^{r_{2}}\left(\frac{\partial}{\partial r_{2}}\right)^{k+l} \mathbb{Q}_{j+1}\left(r_{1}, \xi, \lambda_{i}\right)\left(r_{1}-r\right)^{j+l+k} d r .
\end{aligned}
$$

Each term of the right-hand side of the above expression is $C^{\infty}$ in $r_{1}, r_{2}$ except possibly the last term. But, after applying $\left(\partial / \partial r_{1}\right)^{k}\left(\partial / \partial r_{2}\right)^{l}$ to it then taking the limit $r_{2} \rightarrow r_{1}$ we will get const $\cdot\left(\partial / \partial r_{2}\right)^{k+l} \mathbb{Q}_{j+1}$ which shows $\left(\partial / \partial r_{1}\right)^{k}\left(\partial / \partial r_{2}\right)^{l} \mathfrak{Q}_{j+1}$ exists for $r_{1}=r_{2}$. This completes the proof.

For condition (iii) we need the following lemma. From (2.6) the degree of $\lambda_{i}$ in $a_{j+1}$ increases at most with each increase of index. Therefore $\operatorname{deg} \lambda_{i} a_{j+1} \leqslant j+1$, which we will need in the proof.

II.3. LemMa. $\lim _{t \rightarrow 0} H_{k}\left(r_{1}, x ; \cdot, t\right)=\delta_{(r, x)}$.

Proof. Let $f\left(r_{2}, y\right)$ be a smooth function with compact support which contains the point $\left(r_{1}, x\right)$. Then

$$
\begin{aligned}
& \lim _{t \rightarrow 0} \int_{M \times I} H_{k}\left(r_{1}, x, r_{2}, y ; t\right) f\left(r_{2}, y\right) d V \\
& =\lim _{t \rightarrow 0} \int_{M \times I} H_{k}\left(r_{1}, x, r_{2}, y ; t\right) f\left(r_{2}, y\right) h^{n}\left(r_{2}\right) d r_{2} d y \\
& =\lim _{t \rightarrow 0} \int_{I}\left\{\frac{1}{2 \sqrt{\pi}} h^{-n / 2}\left(r_{1}\right) h^{-n / 2}\left(r_{2}\right) \exp \left(-\frac{\left(r_{1}-r_{2}\right)^{2}}{4 t}\right) t^{-1 / 2}\right. \\
& \cdot \sum_{j=0}^{k}\left[\int_{M_{i=0}}^{\infty} \exp \left(-\frac{\lambda_{i} t}{h\left(r_{1}\right) h\left(r_{2}\right)}\right) \phi_{i}(x) \phi_{i}(y) f\left(r_{2}, y\right)\right. \\
& \left.\left.\cdot a_{j}\left(r_{1}, r_{2}, \lambda_{i}\right) t^{j} d y\right] h^{n}\left(r_{2}\right)\right\} d r_{2} \\
& =\lim _{t \rightarrow 0} \sum_{j=0}^{k} \int_{M_{i}} \sum_{i=0}^{\infty} \exp \left(-\frac{\lambda_{i} t}{h^{2}\left(r_{1}\right)}\right) \phi_{i}(x) \phi_{i}(y) f\left(r_{1}, y\right) a_{j}\left(r_{1}, r_{1}, \lambda_{i}\right) t^{j} d y \\
& =f\left(r_{1}, x\right) \text {. }
\end{aligned}
$$


The second from the last equality holds since $\operatorname{deg}_{\lambda_{i}} a_{j}\left(r_{1}, r_{2}, \lambda_{i}\right)$ is finite. In fact, when $j \neq 0$

$$
\lim _{t \rightarrow 0}\left[\int_{M_{i}} \sum_{i=0}^{\infty} \exp \left(-\frac{\lambda_{i} t}{h_{2}\left(r_{1}\right)}\right) \lambda_{i}^{s} \phi_{i}(x) \phi_{i}(y) f\left(r_{1}, y\right) d y\right] t^{j}=\lim _{t \rightarrow 0} \Delta^{s} f\left(r_{1}, x\right) t^{j}=0
$$

Hence only the term containing $a_{0}=1$ remains. Q.E.D.

For condition (ii) we need the following:

II.4. LEMMA. For given $T>0$

$$
\left|\square H_{k}\right| \leqslant \text { const } t^{[k / 3]-n / 2-3 / 2}, \quad t<T \text {. }
$$

The constant in the above estimate depends on $h(r)$. For the proof of Lemma II.4 some inequalities about the degree of $\lambda_{i}$ in $a_{l}$ are essential. The need to control the degree arises from the fact that each increase in the degree of $\lambda_{i}$ in the expression $\sum_{i=0}^{\infty} \lambda_{i}^{s} e^{-\lambda_{i} t}$ will lower by 1 the degree of $t$ in the asymptotic expression. This follows easily from Theorem I.2.1.

II.5. LEMMA. (i) $\operatorname{deg}_{\lambda_{i}} a_{l}\left(r, r, \lambda_{i}\right) \leqslant[2 l / 3]$.

(ii) $\operatorname{deg}_{\lambda_{i}}\left(\left(\partial / \partial r_{2}\right)^{k} a_{l}\right)\left(r, r, \lambda_{i}\right) \leqslant[(2 l+k-1) / 3], 0<k \leqslant l$.

(iii) $\operatorname{deg}_{\lambda_{i}}\left(\left(\partial / \partial r_{2}\right)^{k} a_{l}\right)\left(r, r, \lambda_{i}\right) \leqslant l, k \geqslant l$.

Proof. By induction on $l$, suppose the above inequalities hold for all $l^{\prime}<l$. Then

$$
\begin{aligned}
& \operatorname{deg}_{\lambda_{i}} a_{l}\left(r, r, \lambda_{i}\right) \leqslant \max \left\{\left[\frac{2 \cdot(l-1)}{3}\right], 1+\left[\frac{2(l-2)}{3}\right]\right. \\
&\left.1+\left[\frac{2(l-2)+1-1}{3}\right], 2+\left[\frac{2(l-3)}{3}\right]\right\} \\
&=\left[\frac{2 l}{3}\right]
\end{aligned}
$$

which proves the first inequality. Consider

$$
\begin{aligned}
{\left[( \frac { \partial } { \partial r _ { 2 } } ) ^ { k } \left(l a_{l}-\left(r_{1}\right.\right.\right.} & \left.\left.\left.-r_{2}\right) \frac{\partial}{\partial r_{2}} a_{l}\right)\right]_{r_{1}=r_{2}} \\
& =\left[l\left(\frac{\partial}{\partial r_{2}}\right)^{k} a_{l}+\left(\begin{array}{c}
k \\
1
\end{array}\right)\left(\frac{\partial}{\partial r_{2}}\right)^{k} a_{l}-\left(r_{1}-r_{2}\right)^{k+1} a_{l}\right]_{r_{1}=r_{2}} \\
& =\left((l+k)\left(\frac{\partial}{\partial r_{2}}\right)^{k} a_{l}\right)\left(r, r, \lambda_{i}\right)
\end{aligned}
$$


and

$$
\begin{aligned}
& l a_{l}-\left(r_{1}-r_{2}\right) \frac{\partial}{\partial r_{2}} a_{l}=-\left(u_{i}\left(r_{2}\right)-\frac{\lambda_{i}}{h\left(r_{1}\right) h\left(r_{2}\right)}\right) a_{l-1}+\left(\frac{\partial}{\partial r_{2}}\right)^{2} a_{l-1} \\
& +\left(r_{1}-r_{2}\right) \frac{\lambda_{i} h^{\prime}\left(r_{2}\right)}{h\left(r_{1}\right) h\left(r_{2}\right)} a_{l-1}+\frac{\lambda_{i}\left(h\left(r_{2}\right) h^{\prime \prime}\left(r_{2}\right)-2\left(h^{\prime}\left(r_{2}\right)\right)^{2}\right)}{h\left(r_{1}\right) h^{3}\left(r_{2}\right)} a_{l-2} \\
& +2 \frac{\lambda_{i} h^{\prime}\left(r_{2}\right)}{h\left(r_{1}\right) h\left(r_{2}\right)^{2}} \frac{\partial}{\partial r_{2}} a_{l-2}+\left(\frac{\lambda_{i} h^{\prime}\left(r_{2}\right)}{h\left(r_{1}\right) h\left(r_{2}\right)}\right)^{2} a_{l-3} .
\end{aligned}
$$

If we want to know the degree of $\lambda_{i}$ in $\left(\left(\partial / \partial r_{2}\right)^{k} a_{l}\right)\left(r, r, \lambda_{i}\right)$ we need only know the degree of the $k$ th derivation w.r.t. $r_{2}$ of the r.h.s. of the above expression:

$$
\begin{aligned}
\left(\frac{\partial}{\partial r_{2}}\right)^{k}\left[\left(u_{i}\left(r_{2}\right)-\right.\right. & \left.\left.\frac{\lambda_{i}}{h\left(r_{1}\right) h\left(r_{2}\right)}\right) a_{l-1}\right]=\left(u_{i}\left(r_{2}\right)-\frac{\lambda_{i}}{h\left(r_{1}\right) h\left(r_{2}\right)}\right)\left(\frac{\partial}{\partial r_{2}}\right)^{k} a_{l-1} \\
& +\left(\begin{array}{c}
k \\
1
\end{array}\right) \frac{\partial}{\partial r_{2}}\left(u_{i}\left(r_{2}\right)-\frac{\lambda_{i}}{h\left(r_{1}\right) h\left(r_{2}\right)}\right)\left(\frac{\partial}{\partial r_{2}}\right)^{k-1} a_{l-1} \\
& +\cdots+\left(\begin{array}{c}
k \\
m
\end{array}\right)\left(\frac{\partial}{\partial r_{2}}\right)^{m}\left(u_{i}\left(r_{2}\right)-\frac{\lambda_{i}}{h\left(r_{1}\right) h\left(r_{2}\right)}\right)\left(\frac{\partial}{\partial r_{2}}\right)^{k-m} a_{l-1} \\
& +\cdots+\left(\frac{\partial}{\partial r_{2}}\right)^{k}\left(u_{i}\left(r_{2}\right)-\frac{\lambda_{i}}{h\left(r_{1}\right) h\left(r_{2}\right)}\right) a_{l-1} .
\end{aligned}
$$

Therefore

$$
\begin{aligned}
& \operatorname{deg}_{\lambda_{i}}\left(\frac{\partial}{\partial r_{2}}\right)^{k}\left[\left(u_{i}\left(r_{2}\right)-\frac{\lambda_{i}}{h\left(r_{1}\right) h\left(r_{2}\right)}\right) a_{l-1}\right]_{r_{1}=r_{2}} \\
& \begin{aligned}
(2.12) \leqslant & \max \left\{1+\left[\frac{2(l-1)+k-1+1}{3}\right], \ldots, 1\right. \\
& \left.+\left[\frac{2(l-1)+k-m-1}{3}\right], \ldots, 1+\left[\frac{2(l-1)}{3}\right]\right\} \\
& =\left[\frac{2 l+k-1}{3}\right] .
\end{aligned}
\end{aligned}
$$


Similarly we have

$$
\begin{gathered}
\operatorname{deg}_{\lambda_{i}}\left(\left(\frac{\partial}{\partial r_{2}}\right)^{k}\left(\frac{\partial}{\partial r_{2}}\right) a_{l-1}\right)_{r_{1}=r_{2}} \leqslant\left[\frac{2 l+k-1}{3}\right], \\
\operatorname{deg}_{\lambda_{i}}\left\{\left(\frac{\partial}{\partial r_{2}}\right)^{k}\left[\left(r_{1}-r_{2}\right) \frac{\lambda_{i} h^{\prime}\left(r_{2}\right)}{h\left(r_{1}\right) h\left(r_{2}\right)} a_{l-1}\right]\right\}_{r_{1}=r_{2}} \leqslant\left[\frac{2 l+k-1}{3}\right], \\
\operatorname{deg}_{\lambda_{i}}\left\{\left(\frac{\partial}{\partial r_{2}}\right)^{k}\left[\frac{\lambda_{i}\left(h\left(r_{1}\right) h^{\prime \prime}\left(r_{2}\right)-2\left(h^{\prime}\left(r_{2}\right)\right)^{2}\right)}{h\left(r_{1}\right)^{3}\left(r_{2}\right)} a_{l-2}\right]\right\}_{r_{1}=r_{2}} \leqslant\left[\frac{2 l+k-1}{3}\right], \\
\operatorname{deg}_{\lambda_{i}}\left\{\left(\frac{\partial}{\partial r_{2}}\right)^{k}\left(\left(\frac{\lambda_{i} h^{\prime}\left(r_{2}\right)}{h\left(r_{1}\right) h^{2}\left(r_{2}\right)}\right) \frac{\partial}{\partial r_{2}} a_{l-2}\right)\right\}_{r_{1}=r_{2}} \leqslant\left[\frac{2 l+k-1}{3}\right], \\
\operatorname{deg}_{\lambda_{i}}\left\{\left(\frac{\partial}{\partial r_{2}}\right)\left(\left(\frac{\lambda_{i} h^{\prime}\left(r_{2}\right)}{h\left(r_{1}\right) h^{2}\left(r_{2}\right)}\right)^{2} a_{l-3}\right)\right\}_{r_{1}=r_{2}} \leqslant\left[\frac{2 l+k-1}{3}\right] .
\end{gathered}
$$

Combining the above inequalities we get

$$
\operatorname{deg}_{\lambda_{i}}\left(\left(\frac{\partial}{\partial r_{2}}\right)^{k} a_{l}\right)\left(r, r, \lambda_{i}\right) \leqslant\left[\frac{2 l+k-1}{3}\right]
$$

so we have proved (ii). (iii) is trivial since $\operatorname{deg}_{\lambda_{i}} a_{l}\left(r_{1}, r_{2}, \lambda_{i}\right)$ is never greater than $l$. Q.E.D.

Now let us return to the proof of Lemma II.4. Consider that (2.15)

$$
\begin{aligned}
& \square_{2} H_{k}= \sum_{i=0}^{\infty}\left[\left(r_{1}-r_{2}\right) \frac{\partial}{\partial r_{2}} a_{k+1}\left(r_{1}, r_{2}, \lambda_{i}\right)-(k+1) a_{k+1}\left(r_{1}, r_{2}, \lambda_{i}\right)\right] G_{i} t^{k-1 / 2} \\
&+\sum_{i=0}^{\infty}\left[\frac{\lambda_{i}\left(h\left(r_{2}\right) h^{\prime \prime}\left(r_{2}\right)-2\left(h^{\prime}\left(r_{2}\right)\right)^{2}\right)}{h\left(r_{1}\right) h\left(r_{2}\right)^{3}} a_{k}\left(r_{1}, r_{2}, \lambda_{i}\right)\right. \\
&-2 \frac{\lambda_{i} h^{\prime}\left(r_{2}\right)}{h\left(r_{1}\right) h^{2}\left(r_{2}\right)} \frac{\partial}{\partial r_{2}} a_{k}\left(r_{1}, r_{2}, \lambda_{i}\right)-\left(\frac{\lambda_{i} h^{\prime}\left(r_{2}\right)}{h\left(r_{1}\right) h^{2}\left(r_{2}\right)}\right)^{2} \\
&\left.\left.+\sum_{i=0}^{\infty}\left[-\left(\frac{\lambda_{i} h^{\prime}\left(r_{2}\right)}{h\left(r_{1}\right) h^{2}\left(r_{2}\right)}\right)^{2} a_{k}\left(r_{1}, r_{2}, \lambda_{i}\right)\right] G_{i} t^{k+3 / 2}, r_{2}, \lambda_{i}\right)\right] G_{i} t^{k+1 / 2}
\end{aligned}
$$

where

$$
G_{i}=\frac{1}{2 \sqrt{\pi}} h^{-n / 2}\left(r_{1}\right) h^{-n / 2}\left(r_{2}\right) \exp \left(-\frac{\left(r_{1}-r_{2}\right)^{2}}{4 t}\right) \exp \left(-\frac{\lambda_{i} t}{h\left(r_{1}\right) h\left(r_{2}\right)}\right) \phi_{i}(x) \phi_{i}(y) .
$$


Since we know that

$$
\begin{array}{cc}
\sum_{i=0}^{\infty} e^{-\lambda_{i} t} \phi_{i}(x) \phi_{i}(y) \leqslant \mathrm{const} \cdot t^{-n / 2}, & t<T, \\
\sum_{i=0}^{\infty} \lambda_{i}^{s} e^{-\lambda_{i} t} \phi_{i}(x) \phi_{i}(y) \leqslant \mathrm{const} \cdot t^{-n / 2-s}, & t<T,
\end{array}
$$

and

$$
\left|\exp \left(\frac{\left(r_{1}-r_{2}\right)^{2}}{4 t}\right)\left(r_{2}-r_{1}\right)^{k}\right| \leqslant \text { const } \cdot t^{-k / 2}, \quad k \neq 0,
$$

it is easy to see that

$$
\begin{gathered}
\mid \sum_{i=0}^{\infty} \exp \left(-\frac{\left(r_{1}-r_{2}\right)^{2}}{4 t}\right) \frac{\left(r_{2}-r_{1}\right)^{k}}{k !}\left(\frac{\partial^{k}}{\partial r_{2} k} a_{l}\left(r_{1}, r_{2}, \lambda_{i}\right)\right)_{r_{1}=r_{2}} \\
\cdot \exp \left(-\frac{\lambda_{i} t}{h\left(r_{1}\right) h\left(r_{2}\right)}\right) \phi_{i}(x) \phi_{i}(y) \mid \\
\leqslant \text { const } \cdot t^{n / 2+k / 2-[(2 l+k-1) / 3]} .
\end{gathered}
$$

Now, we expand $a_{l}\left(r_{1}, r_{2}, \lambda_{i}\right)$ into a Taylor series at $r_{2}=r_{1}$ with $r_{2}$ as variable. If we denote the sum of all terms with power of $\left(r_{2}-r_{1}\right)$ greater than $l$ by $\bar{a}_{l}\left(r_{1}, r_{2}, \lambda_{i}\right)$ then

$$
\begin{aligned}
a_{l}\left(r_{1}, r_{2}, \lambda_{i}\right)= & a_{l}\left(r_{1}, r_{1}, \lambda_{i}\right)+\left(r_{2}-r_{1}\right)\left(\frac{\partial}{\partial r_{2}} a_{l}\left(r_{1}, r_{2}, \lambda_{i}\right)\right)_{r_{1}=r_{2}} \\
& +\cdots+\frac{\left(r_{2}-r_{1}\right)^{k}}{k !}\left[\left(\frac{\partial}{\partial r_{2}}\right)^{k} a_{l}\left(r_{1}, r_{2}, \lambda_{i}\right)\right]_{r_{1}=r_{2}} \\
& +\cdots+\frac{\left(r_{2}-r_{1}\right)^{l}}{l} \bar{a}_{l}\left(r_{1}, r_{2}, \lambda_{i}\right) .
\end{aligned}
$$

Note that $\operatorname{deg}_{\lambda_{i}} \bar{a}_{l}\left(r_{1}, r_{2}, \lambda_{i}\right) \leqslant l$; we have pointed this out in the remark following II.2. In order to estimate $G_{i}$, we multiply each term of the Taylor series expansion by $G_{i}$, and then use the above inequalities to obtain

$$
\left|a_{l}\left(r_{1}, r_{2}, \lambda_{i}\right) G_{i}\right| \leqslant \text { const } \cdot t^{-n / 2-[2 l / 3]} \text {. }
$$

See Corollary 1.2.2. Similarly we can estimate $\square_{2} H_{k}$ since we can estimate each term of it.

$$
\begin{aligned}
\left|\square_{2} H_{k}\right| \leqslant & \text { const } \cdot t^{k-1 / 2-n / 2-[2(k+1) / 3]}+\text { const } \cdot t^{k+1 / 2-n / 2-n / 2-[2 k / 3]-1} \\
& + \text { const } \cdot t^{k+1 / 2-n / 2-[2(k-1) / 3]-2}+\text { const } \cdot t^{k+3 / 2-n / 2-[2 k / 3]-2} \\
\leqslant & \text { const } \cdot t^{[k / 3]-n / 2-3 / 2} .
\end{aligned}
$$

This completes the proof of Lemma II.4 and shows that $H_{k}$ does represent a parametrix for the heat kernel on $M \times I$ when $\left[\frac{k}{3}\right]>\frac{n}{2}+\frac{3}{2}$. Since the heat 
kernel is symmetric w.r.t. its variables $\left(r_{1}, x\right),\left(r_{2}, y\right)$, if we represent it as $\sum_{i=0}^{\infty} f_{i}\left(r_{1}, r_{2}, t\right) \phi_{i}(x) \phi_{i}(y)$ we can see that $f_{i}\left(r_{1}, r_{2}, t\right)$ is also symmetric w.r.t. $r_{1}$ and $r_{2}$. For some special functions $h(r)$ (for example; $h(r)=\sin r$ ),

$$
f_{i}\left(r_{1}, r_{2}, t\right) \phi_{i}(x) \phi_{i}(x) \phi_{i}(y)=\sum_{j=0}^{\infty} a_{j}\left(r_{1}, r_{2}, \lambda_{i}\right) G_{i} t^{j-1 / 2}
$$

is convergent for each $i$. Since $G_{i}$ is symmetric w.r.t. $r_{1}$ and $r_{2}$, in these special cases $A_{j}\left(r_{1}, r_{2}, \lambda_{i}\right)$ is also symmetric w.r.t. $r_{1}$ and $r_{2}$. But if we look at the construction of $a_{j}$ more carefully we find that the form of $a_{j}$ in terms of $h(r)$ is universal ${ }^{2}$ so that we get the following

II.6. CoROllaRY. $a_{j}\left(r_{1}, r_{2}, \lambda_{i}\right)$ is symmetric w.r.t. $r_{1}, r_{2}$.

In proving $H_{k}$ represents a parametrix we also get the following asymptotic expansion:

II.7. Corollary.

$$
\text { Trace } E \underset{t \rightarrow 0}{\sim} \sum_{i=0}^{\infty} \frac{1}{2 \sqrt{\pi}} h^{-n}(r) \sum_{i=0}^{\infty} \exp \left(-\frac{\lambda_{i} t}{h^{2}(r)}\right) a_{j}\left(r, r, \lambda_{i}\right) \phi_{i}^{2}(x) t^{j-1 / 2} .
$$

III. An explicit formula for the asymptotic expansion. In this section we relate the result of §II to the result of Gel'fand and Dikii [4] in order to get an explicit formula. They have studied the asymptotic expansion of the resolvent kernel for the Sturm-Liouville equation and obtained recursive formulas for the coefficients of the expansion. Moreover they also have a generating function for the recursive formulas. Let us start with some formal considerations.

Consider $E\left(r_{1}, r_{2}, x, y, t\right)=\sum_{i=0}^{\infty} f_{i}\left(r_{1}, r_{2}, t\right) \phi_{i}(x) \phi_{i}(y)$ since the Laplace transform of the heat kernel is the kernel of the resolvent of $\Delta[5]$.

$$
\int_{0}^{\infty} e^{-\Delta t} e^{-s t} d t=(\Delta+s)^{-1}
$$

the Laplace transform of each $f_{i}$, should satisfy the following differential equation:

$$
\left[-\left(\frac{\partial}{\partial r_{2}}\right)^{2}-\frac{n h^{\prime}\left(r_{2}\right)}{h\left(r_{2}\right)} \frac{\partial}{\partial r_{2}}+\frac{\lambda_{i}}{h^{2}\left(r_{2}\right)}+s\right] \hat{f}_{i}\left(r_{1}, r_{2}, s\right)=0
$$

or

$$
\begin{gathered}
{\left[-\left(\frac{\partial}{\partial r_{2}}\right)^{2}+\frac{n}{2}\left(\frac{n}{2}-1\right) \frac{h^{\prime \prime}\left(r_{2}\right)^{2}}{h^{2}\left(r_{2}\right)}+\frac{n}{2} \frac{h^{\prime \prime}\left(r_{2}\right)}{h\left(r_{2}\right)}+\frac{\lambda_{i}}{h^{2}\left(r_{2}\right)}\right] h^{n / 2}\left(r_{2}\right) \hat{f}\left(r_{1}, r_{2}, s\right)} \\
=-s h^{n / 2}\left(r_{2}\right) \hat{f}\left(r_{1}, r_{2}, s\right) .
\end{gathered}
$$

\footnotetext{
${ }^{2} a_{j}$ is the summation of $\left(r_{1}-r_{2}\right)^{-1}$ to some power multiplied by the integral of a rational function of $h(r), h^{\prime}(r), \ldots$; the coefficients of the denominator and numerator of the rational function are independent of $h(r)$. See (2.5) for $j=2$.
} 
This in turn suggests that $h^{n / 2}\left(r_{1}\right) h^{n / 2}\left(r_{2}\right) \hat{f}_{i}\left(r_{1}, r_{2}, s\right)$ is the kernel of the resolvent of $-d^{2} / d x^{2}+\left(u_{i}(r)+s\right)$ where $u_{i}(r)$ is defined as in (2.3).

Applying the result of Gel'fand and Dikii [4] we have

$$
h^{n}(r) \hat{f}_{i}(r, r, s) \underset{s \rightarrow \infty}{\sim} \sum_{l=0}^{\infty} \frac{R_{l}\left[u_{i}\right]}{s^{l+1 / 2}}
$$

where $R_{l}=R_{l}\left[u, u^{\prime}, u^{\prime \prime}, \ldots\right]$ is a polynomial in $u, u^{\prime}, u^{\prime \prime}, \ldots$ and satisfies

$$
\begin{gathered}
R_{0}=\frac{1}{2}, \quad R_{1}=-\frac{1}{4} u, \\
4 R_{l+1}=2 \sum_{k=0}^{l-1} R_{k} R_{l-k}^{\prime \prime}-\sum_{k=1}^{l-1} R_{k}^{\prime} R_{l-k}^{\prime} \\
-4 u \sum_{k=0}^{l} R_{k} R_{l-k}-4 \sum_{k=1}^{l} R_{k} R_{l-k+1} .
\end{gathered}
$$

From I.2.3 we see that if $f_{i}$ has an asymptotic expansion it is in the following form:

$$
h^{n}(r) f_{i}(r, r, t) \underset{t \rightarrow 0}{\sim} \sum_{l=0}^{\infty} \frac{R_{l}\left[u_{i}\right]}{\Gamma\left(l+\frac{1}{2}\right)} t^{l-1 / 2} .
$$

The following proposition tells us that it is possible to factor $e^{-u_{i} t}$ out of the above asymptotic expansion.

III.1. Proposition. Summing up those terms in $\sum_{l=0}^{\infty}\left(R_{l}\left[u_{i}\right] / \Gamma\left(l+\frac{1}{2}\right)\right) t^{l-1 / 2}$ which contain a fixed monomial of derivatives of $u_{i}$ of the form $u_{i}^{\left(k_{1}\right)} u_{i}^{\left(k_{2}\right)} \cdots u_{i}^{\left(k_{j}\right)}, k_{j} \neq 0$, gives the sum as $A_{k_{1} \cdots k_{j}} e^{-u_{i} t} u_{i} \cdots u_{i}^{\left(k_{j}\right)} t^{k}, A_{k_{1} \cdots k_{j}}$ is a constant, $k$ is the power of $t$ in the first monomial of such a form.

Proof. From (3.4) a straightforward proof by induction shows (cf. [3]) $\left(\partial / \partial u_{i}\right) R_{l}\left[u_{i}\right]=-\left(l-\frac{1}{2}\right) R_{l-1}\left[u_{i}\right]$ which in turn implies

$$
\frac{\partial}{\partial u_{i}} \frac{R_{l}\left[u_{i}\right]}{\Gamma\left(l+\frac{1}{2}\right)}=-\frac{R_{l-1}\left[u_{i}\right]}{\Gamma\left(l-1+\frac{1}{2}\right)} \text {. Q.E.D. }
$$

If we compare the expression after factoring $e^{-u_{i} t}$ out of (3.5) with the asymptotic expansion in the end of the last section, we find that we should multiply some part of $e^{-u_{i} t}$ back into the expression. Split $u_{i}$ into the following two parts:

$$
u_{i}(r)=q(r)+\lambda_{i} / h^{2}(r) .
$$

Then multiplying $e^{-q(r) t}$ back into the expression, we obtain

$$
f_{i}(r, r, t) \underset{t \rightarrow 0}{\sim} \frac{1}{2 \sqrt{\pi}} h^{-n / 2}(r) \exp \left(-\frac{\lambda_{i} t}{h^{2}(r)}\right) \sum_{j=0}^{\infty} \tilde{a}_{j}\left(r, \Lambda_{i}\right) t^{j-1 / 2} .
$$

Therefore $\tilde{a}_{j}\left(r, \lambda_{i}\right)$ is nothing but $\left(R_{j}\left[q, u_{i}^{\prime}, u_{i}^{\prime \prime}, \ldots\right] / \Gamma\left(j+\frac{1}{2}\right)\right) \cdot 2 \sqrt{\pi}$, i.e. replace $u_{i}$ (but not its derivatives) in $R_{j}$ by $q$.

The following theorem is essential for the purpose of this section.

III.2. THEOREM. $a_{j}\left(r, r, \lambda_{i}\right)=\tilde{a}_{j}\left(r, \lambda_{i}\right)$. 
Proof. Consider the expression

$$
\begin{aligned}
K_{1}\left(r_{1}, r_{2}, \lambda_{i}\right)= & u_{i}\left(r_{2}\right)\left[a_{l-1}-a_{l-2} \frac{\lambda_{i}}{h\left(r_{1}\right) h\left(r_{2}\right)}+\frac{1}{2 !} a_{l-3}\left(\frac{\lambda_{i}}{h\left(r_{1}\right) h\left(r_{2}\right)}\right)^{2}\right. \\
& \left.+\cdots+(-1)^{l-1} \frac{1}{(l-1) !}\left(\frac{\lambda_{i}}{h\left(r_{1}\right) h\left(r_{2}\right)}\right)^{l-1} a_{0}\right] \\
& +l\left[a_{l}-a_{l-1} \frac{\lambda_{i}}{h\left(r_{1}\right) h\left(r_{2}\right)}+\cdots+(-1)^{l} \frac{1}{l !}\left(\frac{\lambda_{i}}{h\left(r_{1}\right) h\left(r_{2}\right)}\right)^{l} a_{0}\right] \\
& +\left(r_{1}-r_{2}\right) \frac{\partial}{\partial r_{2}}\left[a_{l}-a_{l-1} \frac{\lambda_{i}}{h\left(r_{1}\right) h\left(r_{2}\right)}\right. \\
& \left.+\frac{\partial}{\partial r_{2}}\left[a_{l-1}-a_{l-2}\left(\frac{\lambda_{i}}{h\left(r_{1}\right) h\left(r_{2}\right)}\right)^{l}\right)^{a_{0}}\right] \\
& +\cdots+(-1)^{l} \frac{1}{(l-1) !}\left(\frac{\lambda_{i}}{h\left(r_{1}\right) h\left(r_{2}\right)}\left(\frac{\lambda_{i}}{h\left(r_{1}\right) h\left(r_{2}\right)}\right)^{l-1} a_{0}\right]
\end{aligned}
$$

We can express the right-hand side of the above as the sum of terms of the following form:

$$
\begin{aligned}
(-1)^{k} \frac{1}{k !}\left(\frac{\lambda_{i}}{h\left(r_{1}\right) h\left(r_{2}\right)}\right)^{k}[ & \left(u_{i}\left(r_{2}\right)-\frac{\lambda_{i}}{h\left(r_{1}\right) h\left(r_{2}\right)}\right) a_{l-k-1}-\left(\frac{\partial}{\partial r_{2}}\right)^{2} a_{l-k-1} \\
& \quad\left(r_{1}-r_{2}\right) \frac{\lambda_{i}}{h\left(r_{1}\right)}\left(\frac{1}{h\left(r_{2}\right)}\right)^{\prime} a_{l-k-1}+(l-k) a_{l-k} \\
& \quad-\left(r_{1}-r_{2}\right) \frac{\partial}{\partial r_{2}} a_{l-k}+\frac{\lambda_{i}}{h\left(r_{2}\right)}\left(\frac{\lambda_{i}}{h\left(r_{2}\right)}\right)^{\prime \prime} a_{l-k-2} \\
+ & \left.\frac{2 \lambda_{i} i}{h\left(r_{1}\right)}\left(\frac{1}{h\left(r_{2}\right)}\right)^{\prime} \frac{\partial}{\partial r_{2}} a_{l-k-2}-\frac{\lambda_{i}}{h\left(r_{1}\right)}\left(\left(\frac{1}{h\left(r_{2}\right)}\right)^{\prime}\right)^{2} a_{l-k-3}\right] .
\end{aligned}
$$

Since $a_{l}$ satisfies (2.2) of the last section, each term of this form must be zero and so is their sum, i.e. $K_{1}\left(r_{1}, r_{2}, \lambda_{i}\right)=0$. Similarly, if we denote by $K_{2}\left(r_{1}, r_{2}, \lambda_{i}\right)$ the expression which comes from $K_{1}\left(r_{1}, r_{2}, \lambda_{i}\right)$ by interchanging the roles played by $r_{1}$ 
and $r_{2}$, then we also have $K_{2}\left(r_{1}, r_{2}, \lambda_{i}\right)=0$. Moreover,

$$
\begin{array}{ll}
\frac{\partial}{\partial r_{2}} K_{1}\left(r_{1}, r_{2}, \lambda_{i}\right)=0, & 3 \frac{\partial}{\partial r_{1}} K_{1}\left(r_{1}, r_{2}, \lambda_{i}\right)=0, \\
3 \frac{\partial}{\partial r_{2}} K_{2}\left(r_{1}, r_{2}, \lambda_{i}\right)=0, & \frac{\partial}{\partial r_{1}} K_{2}\left(r_{1}, r_{2}, \lambda_{i}\right)=0 .
\end{array}
$$

Combining these identities, and letting $r_{1}=r_{2}$, we get

$$
\begin{aligned}
\left(l-\frac{1}{2}\right)\left[a_{l}-\right. & \left.\frac{\lambda_{i}}{h^{2}} a_{l-1}+\frac{1}{2}\left(\frac{\lambda_{i}}{h^{2}}\right)^{2} a_{l-2}+\cdots\right]^{\prime} \\
= & \frac{1}{4}\left[a_{l-1}-a_{l-1} \frac{\lambda_{i}}{h^{2}}+\frac{1}{2 !}\left(\frac{i}{h^{2}}\right)^{2} a_{l-3}+\cdots\right]^{\prime \prime \prime} \\
& -\frac{1}{2} u_{i}^{\prime}\left[a_{l-1}-a_{l-2}-\frac{\lambda_{i}}{h^{2}}+\ldots\right]-u_{i}\left[a_{l-1}-a_{l-2} \frac{\lambda_{i}}{h^{2}}+\cdots\right]
\end{aligned}
$$

Now, since by construction of $\tilde{a}_{l}$ we have

$$
\left[\tilde{a}-\tilde{a}_{l-1} \frac{\lambda_{i}}{h^{2}}+\cdots+\frac{(-1)^{l}}{l !} a_{0}\left(\frac{\lambda_{i}}{h^{2}}\right)^{l}\right]=\frac{R_{l}}{\Gamma\left(l+\frac{1}{2}\right)} 2 \sqrt{\pi}
$$

and from (3.4) by induction we have $R_{l}^{\prime}=\frac{1}{4} R_{l-1}-u R_{l-1}^{\prime}-\frac{1}{2} u^{\prime} R_{l-1}$, then by substitution we will see that $a_{l}$ satisfies the same differential equation as above. Since both of them satisfy the same differential equation of first order they may differ by a constant. But because this constant is universal, ${ }^{3}$ it is sufficient to check the special case $h=$ const. Then we will get that it must be zero, so that $\tilde{a}_{j}\left(r, r, \lambda_{i}\right)=\tilde{a}_{j}\left(r, \lambda_{i}\right)$. Q.E.D.

\section{III.3. COROLlary.}

$$
\text { Trace } E \underset{t \rightarrow 0}{\sim} \sum_{j=0}^{\infty} \frac{1}{\sqrt{4 \pi}} h^{-n}(r) \sum_{i=0}^{\infty} \exp \left(-\frac{\lambda_{i} t}{h^{2}(r)}\right) \tilde{a}_{j}\left(r, \lambda_{i}\right) \phi_{i}^{2}(x) t^{j-1 / 2} .
$$

This proves the legitimacy of the addition of the infinitely many asymptotic expansions to form the asymptotic expansion we wanted.

Now we are ready to calculate the coefficients. Now if we let

$$
\begin{aligned}
\sum_{i=0}^{\infty} e^{-\lambda_{i} t} \phi_{i}^{2}(x) \underset{t \rightarrow 0}{\sim} \frac{1}{\sqrt{4 \pi}^{n}}\left(C_{0} t^{-n / 2}\right. & +C_{1} t^{-n / 2} \\
& \left.+\cdots+C_{s} t^{-n / 2}+\cdots+C_{s} t^{-n / 2+s}+\cdots\right)
\end{aligned}
$$

\footnotetext{
${ }^{3}$ From the constructions of both $a_{l}$ and $\tilde{a}_{l}$ we see that the coefficients involved in them are independent of $h(r)$.
} 
then

$$
\begin{aligned}
\sum_{i=0}^{\infty} \lambda_{i}^{l} e^{-\lambda_{i} t} \phi_{i}^{2}(x) \underset{t \rightarrow 0}{\sim} \frac{1}{\sqrt{4 \pi}^{n}}[ & C_{0}\left(\frac{n}{2}\right)\left(\frac{n}{2}+1\right) \cdots\left(\frac{n}{2}+l-1\right) t^{-n / 2-l} \\
& +C_{1}\left(\frac{n}{2}-1\right) \frac{n}{2} \cdots\left(\frac{n}{2}+l-2\right) t^{-n / 2-l+1} \\
& \left.+\cdots+C_{s}\left(\frac{n}{2}-s\right) \cdots\left(\frac{n}{2}+l-s\right) t^{-n / 2-l+s}+\cdots\right]
\end{aligned}
$$

where $l \geqslant 1$ and

$$
\begin{aligned}
& \sum_{i=0}^{\infty} \lambda_{i} \exp \left(-\frac{\lambda_{i} t}{h^{2}(r)}\right) \phi_{i}^{2}(x) \\
& \underset{t \rightarrow 0}{\sim} \frac{1}{\sqrt{4 \pi}^{n}}\left[C_{0}\left(\frac{n}{2}\right)\left(\frac{n}{2}+1\right) \cdots\left(\frac{n}{2}+l-s\right)\left(\frac{t}{h^{2}(r)}\right)^{-n / 2-l}\right. \\
&+\cdots+C_{s}\left(\frac{n}{2}-s\right) \cdots\left(\frac{n}{2}+l-s\right) \\
&\left.+\left(\frac{t}{h^{2}(r)}\right)^{-n / 2-l+s}+\cdots\right]
\end{aligned}
$$

Suppose we denote the coefficients of $\lambda_{i}^{l}$ in $\tilde{a}_{j}^{l}\left(r, \lambda_{i}\right)$ by $a_{j}^{l}(r)$, and then consider those terms containing $t^{-(n+1) / 2+k}$ which come from $\tilde{a}_{j}\left(r, \lambda_{i}\right)$.

$$
\frac{1}{\sqrt{4 \pi}} h^{-n}(r) \frac{1}{\sqrt{4 \pi}} a_{j}^{l}(r) C_{s}^{l}\left(\frac{n}{2}-s\right) \cdots\left(\frac{n}{2}+l-s\right)\left(\frac{t}{h^{2}(r)}\right)^{n / 2+s-l} t^{j-1 / 2}
$$

with $-l+s+j=k$. But since $\operatorname{deg}_{\lambda_{i}} \tilde{a}_{j}=\operatorname{deg}_{\lambda_{i}} a_{j}\left(r, r, \lambda_{i}\right) \leqslant[2 j / 3]$ and $s \geqslant 0$ then $j \leqslant 3 k$. Now let $j$ vary and collect all those terms containing $t^{-(n+1) / 2+k}$. We will have

$$
\begin{aligned}
\sum_{j=0}^{[2 j / 3]} \sum_{j=0}^{3 k} \frac{1}{\sqrt{4 \pi t}^{n+1}} a_{j}^{l} C_{k-j+1}\left(\frac{n}{2}-k+j-l\right) \cdots \\
\\
\quad\left(\frac{n}{2}-k+j-1\right)\left(\frac{t}{h^{2}(r)}\right)^{k} h^{2 j}(r) .
\end{aligned}
$$

If we denote

$$
d_{k}=\sum_{l=0}^{[2 j / 3]} \sum_{j=0}^{3 k} a_{j}^{l} C_{k-j+1}\left(\frac{n}{2}-k+j-l\right) \cdots\left(\frac{n}{2}-k+j-1\right) h^{2 j}(r)
$$


then

$$
\text { Trace } E \underset{t \rightarrow 0}{\sim} \sum_{k=0}^{\infty} \frac{1}{\sqrt{4 \pi t}^{n+1}} d_{k}\left(\frac{1}{h^{2}(r)}\right)^{k} .
$$

REMARK. When $l=0,\left(\frac{n}{2}-k+j-l\right) \cdots\left(\frac{n}{2}-k+j-1\right)$ will be replaced by 1 .

IV. The significance of the approach and applications of the result. In the end of the last section we got a formula for the coefficients $d_{k}$ on $M \times I$ in terms of coefficients $c_{j}$ and $a_{j}^{l}$ where $a_{j}^{l}$ are the coefficients of $\lambda_{i}^{l}$. When we write $a_{j}$ into the polynomial of $\lambda_{i}$, we should know $a_{j}$. Although the $a_{j}$ can be constructed by the recursive formula which we have derived in $\$ I I$, a generating function for them will be more helpful. Gel'fand and Dikii have a generating function for $R_{l}$ which can be exploited to construct $a_{l}$ as a polynomial in $u, u^{\prime}, u^{\prime \prime}, \ldots$ and then to translate $R_{l}$ into the so-called symbolic polynomial, which in turn has a generating function. Judging from the sophisticated way they derived the function, we expect that a generating or even a more directly recursive formula for $d_{k}$ than the one we got is unlikely.

Now we will give some arguments to show why we cannot start from (0.3), the standard parametrix, and get a reasonable result. Let us consider first the case of the metric cone, $h(r)=r$. Since the first coefficient of the asymptotic expansion of the heat kernel is the reciprocal of the square root of the determinant of the exponential map, we should find the relation between the determinant of the exponential map on the cone and the determinant of the exponential map on the base. ${ }^{4}$

A straightforward calculation leads us to

$$
\theta((p, \tau),(Q, \kappa))=\underline{\theta}(P, Q)\left(\frac{l}{\sin l}\right)^{n-1}
$$

where $\theta((p, \tau),(Q, \kappa))$ denotes the determinant of the exponential map from tangent space $M \times I_{(p, \tau)}$ to $M \times I$ evaluated at $\left(\exp _{(p, \tau)}\right)^{-1}(Q, \kappa), \underline{\theta}(P, Q)$ denotes the corresponding notion on $M$, and $l$ denotes the distance between $P$ and $Q$ on $M$. In order to find the coefficients of the asymptotic expansion of the heat kernel we should apply $\Delta$ to $\theta^{-1 / 2}\left[1\right.$, p. 208]. From the relation $\Delta=\Delta / r^{2}-(n / r)(\partial / \partial r)-$ $\partial^{2} / \partial r^{2}$ and the fact that the right-hand side of the above relation is independent of $r$ we have

$$
\begin{aligned}
\Delta^{k} \theta^{-1 / 2}=\frac{\Delta}{r^{2 k}}(\Delta+2 n-2.3)(\Delta+4 n-4.5) & \\
& \cdots(\Delta+(2 k-2) n-(2 k-2)(2 k-1)) \theta^{-1 / 2}\left(\frac{l}{\sin l}\right)^{-(n-1) / 2} .
\end{aligned}
$$

This formula shows the complication of a direct computation. There is the difficulty not only of writing out explicitly the operator in the right-hand side into a

\footnotetext{
${ }^{4}$ More generally, the $k$ th coefficients are something like $\Delta^{k} \theta^{-1 / 2}$ on the trace (i.e. $\left.r_{1}=r_{2}, x=y\right)[1, p$ 208].
} 
polynomial of $\Delta$ but also of applying each term to a product of two functions. Moreover the expression does not lead immediately to the formula of correct type, i.e. coefficients on $M \times I$ are a linear combination of coefficients on base $M$. Thus our result implies some interesting cancellation. In the case of more general $h(r)$ it becomes even more hopeless since the relation between the determinant of the exponential maps must involve $r$. This involvement of $r$ will make the application of $\Delta$ to the determinant even more complicated and it will be unlikely there is any expression as the above one.

For the first application, if we set $h(r)=r$ and $d_{k}=0$ in formula (3.19) this will give us a recursive formula for $C_{j}$ which is just the coefficient of the asymptotic expansion of the trace of the heat kernel on $S^{n}$. The formula so obtained is no more complicated than the corresponding formula obtained by Cahn and Wolf [2]. In their formula they have two cases, when $n$ is even or odd. We have a single formula for all $n$. However their approach is more general and they apply it to the cases of compact symmetric spaces of rank one.

For the second application, let us consider the special case when $M$ is flat. In this case all the coefficients $c_{i}$ in the expansion for trace of the heat kernel of $M$ except $c_{0}$ are zero. Then

$$
d_{k}=a_{k+l}^{l}\left(\frac{n}{2}\right)\left(\frac{n}{2}+1\right) \cdots\left(\frac{n}{2}+l-1\right) h^{2(k+l)}(r) .
$$

Here, as before $\left(\frac{n}{2}\right)\left(\frac{n}{2}+1\right) \cdots\left(\frac{n}{2}+l-1\right)$ will be repaced by 1 when $l=0$.

Let us list below the first two nontrivial coefficients:

$$
\begin{aligned}
d_{1}= & -\frac{n(n-1)}{6} h^{\prime}(r)^{2}-\frac{n}{3} h(r) h^{\prime \prime}(r), \\
d_{2}= & \frac{n(n-1)\left(5 n^{2}+17 n-66\right)}{360} h^{\prime}(r)^{4} \\
& -\frac{n\left(n^{2}-36 n+41\right)}{90} h(r) h^{\prime}(r)^{2} h^{\prime}(r) \\
& -\frac{n(2 n-3)}{15} h^{2}(r) h^{\prime}(r) h^{\prime \prime \prime}(r)-\frac{n}{15} h^{3}(r) h^{I v}(r) \\
& -\frac{n(n-9)}{60} h^{2}(r)^{2} h^{\prime \prime}(r)^{2} .
\end{aligned}
$$

In general $d_{k}$ is a polynomial of $h(r)$ and its derivatives with homogeneous differential order $2 k$.

\section{BIBLIOGRAPHY}

1. M. Berger, P. Gauduchon and E. Mazet, Le spectre d'une variété Riemannienne, Lecture Notes in Math. vol. 194, Springer-Verlag, Berlin, Heidelberg and New York, 1971.

2. R. S. Cahn and J. A. Wolf, Zeta function and their asymptotic expansions for compact symmetric space of rank one (preprint).

3. J. Cheeger and S. Yau, A lower bound for the heat kernel (preprint). 
4. I. M. Gel'fand and L. A. Dikii, Asymptotic behavior of the resolvent of Sturm-Liouville equations and the algebra of the Korteweg-de Vries equations, Russian Math. Surveys 30 (1975).

5. P. Gilkey, The index theorem and the heat equation, Publish or Perish, Boston, Mass., 1974.

6. H. Lauwerier, Asymptotic analysis, Mathematical Center Tracts, Math. Centrum, Amsterdam, 1977.

7. S. Minakshisundaram and A. Pleijel, Some properties of the eigenfunctions of the Laplace operator on Riemannian manifold, Canad. J. Math. 1 (1949).

8. S. Minakshisundaram, Eigenfunctions on Riemannian manifolds, J. Indian Math. Soc. 17 (1953).

9. A. Zemanian, Generalized integral transformations, Interscience, New York, 1968.

Department of Mathematics, Purdue University, West LafayetTe, Indiana 47907 